\title{
Searching for a link between the presence of chemical spots on the surface of HgMn stars and their weak magnetic fields
}

\author{
Igor S. Savanov $^{1}$, S. Hubrig ${ }^{2}$, J. F. González ${ }^{3}$ and M. Schöller ${ }^{2}$ \\ ${ }^{1}$ Institute of Astronomy, Russian Academy of Science, Moscow, Russia; \\ email: isavanov@rambler.ru \\ ${ }^{2}$ ESO, Santiago, Chile \& Garching, Germany \\ ${ }^{3}$ Complejo Astronomico El Leoncito, San Juan, Argentina
}

\begin{abstract}
We present the results of mapping the HgMn star AR Aur using the Doppler Imaging technique for several elements and discuss the obtained distributions in the framework of a magnetic field topology.
\end{abstract}

Keywords. Stars: atmospheres - stars: chemically peculiar - stars: magnetic fields - stars: spots

\section{Introduction}

Late B-type stars with $\mathrm{HgMn}$ peculiarity are characterized by low rotational velocities and weak or non-detectable magnetic fields. The most distinctive features of their atmospheres are the extreme overabundance of $\mathrm{Hg}$ and $\mathrm{Mn}$. More than 2/3 of $\mathrm{HgMn}$ stars belong to spectroscopic binaries. The presence of an inhomogeneous distribution of some elements over the surface of HgMn stars was discussed for the first time by Hubrig \& Mathys (1995). The first definitively identified spectrum variability has been reported for the binary star $\alpha$ And by Wahlgren et al. (2001) and Adelman et al. (2002).

\section{Doppler imaging}

We present the first results of our preliminary Doppler Imaging (DI) modeling of abundance distributions for four elements on the surface of the HgMn star AR Aur (Fig. 1). The analysis of the spectra is made with the DI inversion code iAbu, which reconstructs the stellar surface abundance inhomogeneities from the series of spectral line profiles using the Tikhonov regularization algorithm.

Previously, Hubrig et al. (2006) concluded that the employment of a partially fractured equatorial ring presents a realistic distribution of $\mathrm{Y}$ on the stellar surface. Our new map based on the inversion of the Y II $\lambda 4900 \AA$ line is in good agreement with this conclusion. The $\mathrm{Y}$ is overabundant and strongly concentrated in the equatorial ring, where the average $Y$ abundance reaches $\log \varepsilon(Y)=6.0$ (in the scale $\log \varepsilon(H)=12.0)$. One large fraction of the ring is missing exactly on the surface area which is permanently facing the secondary. An additional polar detail can be seen at longitudes $180^{\circ}-270^{\circ}$. The surface distribution of strontium was studied with the ion line Sr II $\lambda 4215 \AA$. The Sr surface distribution in AR Aur is not completely similar to the map reconstructed for Y II, although it also presents significant equatorial details of Sr overabundance in the range of longitudes from $70^{\circ}$ to $250^{\circ}$.

In the case of the $\mathrm{Hg}$ II $\lambda 3984 \AA$ line we normalized our spectra relative to the pseudocontinuum of the $\mathrm{H} \varepsilon$ wing, but accounted for blending by the hydrogen line in the 

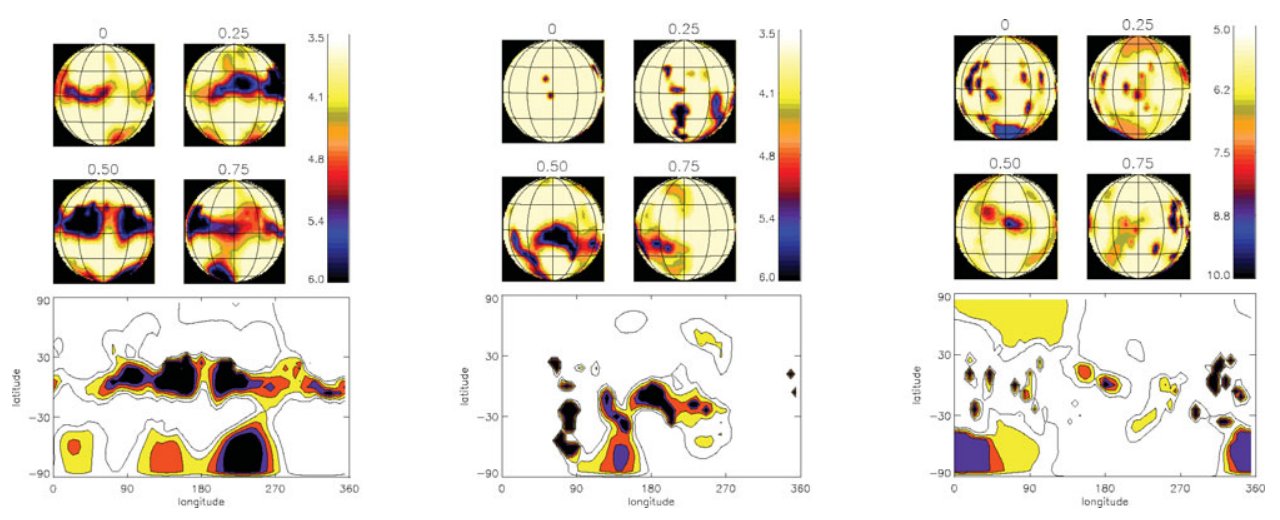

Figure 1. Results of the DI reconstruction of the $\mathrm{Y}, \mathrm{Sr}$ and $\mathrm{Hg}$ surface distributions (from left to right). The rectangular plots (lower panels) show a pseudo-Mercator projection of the surface maps. Spherical DI maps are presented at four equidistant rotational phases (upper panels).

calculations of the synthetic spectra by adding corresponding opacity sources. The line list used for the DI consists of isotopic and hyperfine structure components of the $\mathrm{Hg}$ II $\lambda 3984 \AA$ line and a Y II $\lambda 3982.59 \AA$ blending line. We parameterized the mercury isotope composition using the isotopic-fractionation model of White et al. (1976). Our observational data are insufficient for a simultaneous determination of both the $\mathrm{Hg}$ abundance and the surface distribution of the q-parameter, hence we accept $q=0$ for the terrestrial mixture. The mercury overabundance is strongly concentrated in several equatorial details on the surface of the star. Further we find polar appendages at longitudes $0^{\circ}-90^{\circ}$

For the first time we reconstructed the manganese surface distribution in a HgMn star. The Mn-ion map is based on modeling of the Mn II $\lambda 4292 \AA$ line. This map is not shown in Fig. 1 due to lack of space. We demonstrated that the Mn surface distribution shows similarities with those of $\mathrm{Y}$ and $\mathrm{Sr}$, namely both equatorial and polar features.

\section{Conclusions}

What is the explanation for the discovered inhomogeneities? Taking into account that more than 2/3 of the HgMn stars are known to belong to spectroscopic binaries, a scenario how a magnetic field can be built up in binary systems has been presented some time ago by Hubrig (1998) who suggested that a tidal torque varying with depth and latitude in a star induces differential rotation. Differential rotation in a radiative star can, however, be prone to the magneto-rotational instability (e.g., Arlt et al. 2003). Magneto-hydrodynamical simulations revealed a distinct magnetic field topology similar to the latitudinal fractured rings observed on the surface of $\alpha$ And and AR Aur.

\section{References}

Adelman, S. J., Gulliver, A. F., Kochukhov, O. P., \& Ryabchikova, T. A. 2002, ApJ 575, 449 Arlt, R., Hollerbach, R., \& Rüdiger G. 2003, A\&A 401, 1087

Hubrig, S. \& Mathys, G. 1995, Comments Astrophys. 18, 167

Hubrig, S. 1998, CoSka 27, 296

Hubrig, S., Gonzales, J. F., Savanov, I., Schöller, M., Ageorges, N., Cowley, C. R., \& Wolff, B. 2006, MNRAS 371, 1953

Wahlgren, G. M., Ilyin, I., \& Kochukhov, O. 2001, A\&A 33, 1506

White, R. E., Vaughan, A. H.,Jr., Preston, G. W., \& Swings, J. P. 1976, ApJ 204, 131 